\title{
A Binary Code Learning Method Based on Class- neighborhood for Finger Vein Recognition
}

\author{
Liping Zhang ${ }^{1.2 .3}$, Weijun $\mathrm{Li}^{1,2,3, *}$, Xin Ning ${ }^{1.3}$, Xiaoli Dong ${ }^{1.2 .3}$ and Yakun Zhang ${ }^{1.2 .3}$ \\ ${ }^{1}$ Institute of Semiconductors, Chinese Academy of Sciences, Beijing, 100083, China \\ ${ }^{2}$ School of Microelectronics, University of Chinese Academy of Sciences, Beijing 100029, China \\ ${ }^{3}$ Joint Laboratory for Cognitive Computing Technology, WAVE Group, Beijing 100083, China \\ ${ }^{*}$ Corresponding author
}

\begin{abstract}
The finger vein recognition, as a new biometric identification technology, judges the identity of a person by using their finger vein angiography images obtained by near-infrared light, and has recently drawn considerable attention from scientists worldwide. In this work, a novel binary code learning method based on class-neighborhood (BCLCN) is proposed for finger vein recognition. Different from traditional binary code methods based on pixels, BCLCN fully considers the relationships between categories of train set and encodes based those relationships. In this way, the discrimination of the code is enhanced and the binary codes are shortened. Experimental results on self-built finger vein image database demonstrate the effectiveness of BCLCN $(E E R=0.512 \%)$.
\end{abstract}

Keywords-finger vein recognition; binary code learning; class-neighborhood

\section{INTRODUCTION}

Along with the development of the techniques of artificial intelligence and computer science, traditional identification ways, such as key, password, entrance card are eliminated gradually, replaced by various kinds of biometrics identifier, such as face, finger print, iris, palmprint (human's physical characteristics) and gait, voice, handwriting (human's behavior characteristics), etc. In comparison with traditional identification ways, these biometrics identifiers demonstrate some excellent advantages: convenient, quick, effective, not easily lost, good interaction. Veins are subcutaneous structure that randomly develop into a network, accordingly, finger veins are criss-crossing vein patterns spread along a finger. Medical research shows that, different persons, even the same person's different fingers have different vein distributions, and the distributions will not change with age and environment once formed [1,2]. Finger vein recognition is a biometrics technology based on the criss-crossing vein patterns. To visualize veins in a finger, the near infrared(NIR) lights (690 980nm) are often used in finger-vein image acquisition systems. Compared with other biometric identification technologies, finger vein recognition demonstrates four main advantages in application: (1) non-contact collection: contactless data collection ensures users free to contagion and unpleasant sensations; (2)Vivo identification: finger vein recognition systems are uncheatable by fake finger since finger veins are blood vessel network randomly located inside a finger, accordingly, fake finger attacks seriously in finger print recognition; (3)finger vein patterns are internal features, not visible to the naked eye that are difficult to counterfeit; (4)small size and simple structure of imaging devices: smaller size of finger vein capturing devices makes this biometric identifier more easily be ported to different devices like mobile, tablet PC, etc. Due to the positive features, finger vein recognition has attracted considerable attentions in the biometric recognition field since it was put forward in 2000.

The related work about finger vein recognition earliest began in Japan, and at present the country which has the most mature technique is Japan as well. Many efforts are contributed to develop this technology and the technology has made remarkable progress with these efforts in the resent two decades. The representative methods include repeated line tracking by Miura[3], various feature extraction methods based on Gabor filter[4,5], local binary pattern (LBP)method based on local information[6], band-limited phase only correlation filter (BLPOC)[7], histogram of oriented gradients (HOG) descriptor[8], and various subspace learning methods, etc. These methods investigate various feature description for finger vein.

Most of existing binary code methods focus on representative information of image itself, which have two limitations. On one hand, they ignore the inter-class relationship, while the discriminative information among different classes may be important for describing the subject. On the other hand, the codes generated by existing methods usually have almost the same size as input image because most of them are generated based on each pixel, and the large number of bits leads to storage requirements and increasing time cost.

Reference [9] proposed a finger vein recognition method based on discriminative binary codes learning. Firstly, it constructed subject relation to capture the inter-class correlation. Then binary code templates of subjects were generated according to the subject relationship.

In this paper, we proposed a novel binary code learning method based on class-neighborhood(BCLCN) for finger vein recognition. First, subject relationship matrix is constructed by calculating the distance between different training subjects. Then, k class-neighborhood locations are determined for each class and we can generate the binary code templates according to $\mathrm{k}$ class-neighborhood locations, then information entropy of binary code templates is considered to determine the value of 
k. At last, SVMs are trained as code learner for each bit, with all training samples as input and binary code templates as supervised information. Several groups of experiment are tested to illustrate the effectiveness of the proposed method.

\section{RELATED WORK}

\section{A. Subject Relationship Matrix Construction}

In order to obtain the subject relationship matrix, we should find the centers of different subjects first, only the centers are used to compute the between-class correlation coefficients. In our method, the center of each subject is calculated by averaging all training samples from the subject. Suppose we have $M$ subject, then the relationship matrix has $M \times M$ elements. In this step, the within-class variations are ignored to simplify the relationship matric because we hope to capture the between-class correlation. Then, Euclidean distance between two centers are used to represent the correlation of two subjects.

Figure 1 shows an example of subject relationship matrix, the matrix is constructed by self-built finger vein database, the darker color, the weaker class correlation.



FIGURE I. AN EXAMPLE OF SUBJECT RELATIONSHIP MATRIX.

From figure 1, we can observe that there is exactly discrimination in between-class correlation, some subjects are "close" and some subjects are "far away". To amplify the difference of the correlation, we need further processing with the original relational matrix. First, the relationship matrix is converted to integers between 0 and 255, and it can be treated as an image matrix. Then we use histogram equalization by each row and column to amplify the correlation difference in each subject and bit respectively. Figure 2 and 3 show the correlation changes between pre- treatment and post-treatment.
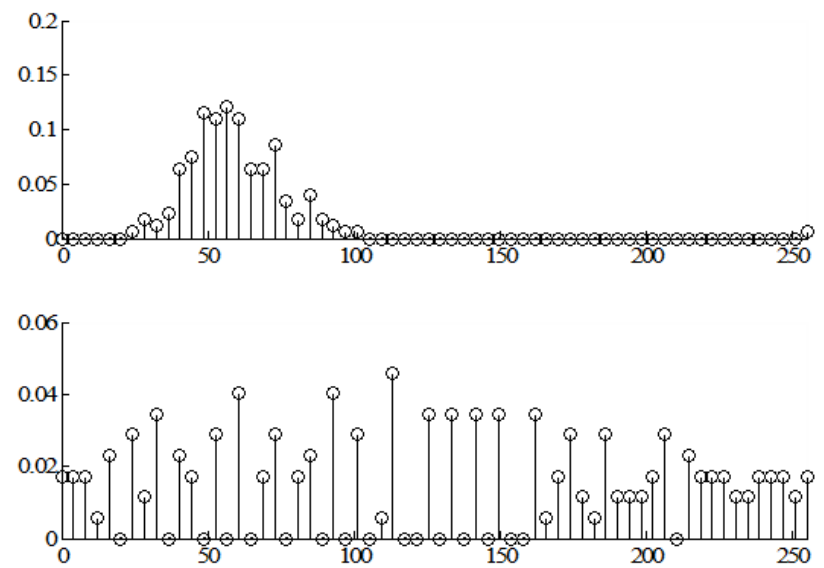

FIGURE II. THE CORRELATION CHANGE IN ONE SUBJECT BETWEEN PRE- TREATMENT AND POST-TREATMENT.
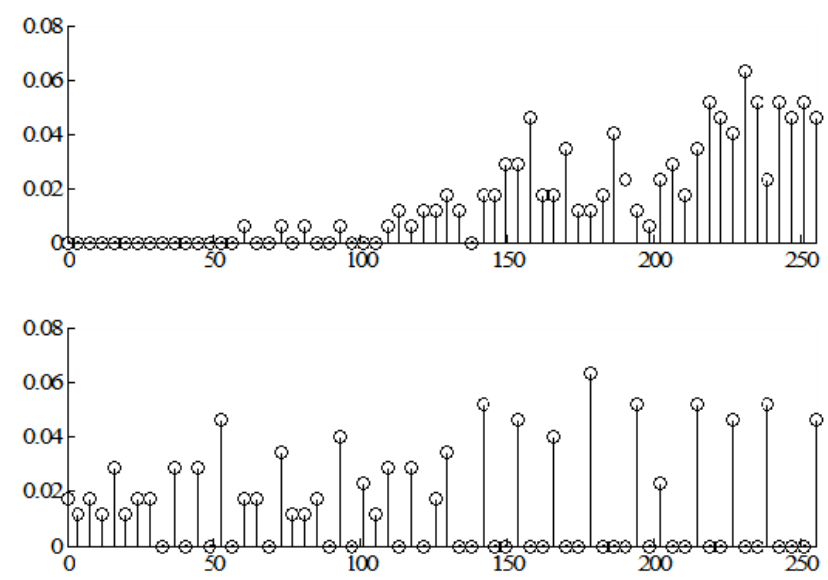

FIGURE III. THE CORRELATION CHANGE IN ONE BIT BETWEEN PRE- TREATMENT AND POST-TREATMENT.

From these two figures, we can observe that the correlations in one subject or one bit are close before equalization, and after equalization, the correlations have better expressive ability.

\section{B. Binary Code Templates Generation}

Based on the subject relationship matrix after histogram equalization, we hope to generate effective binary code templates, because the templates are used to describe the between-class characteristics. Above all, the templates as supervised information of all training samples will be input to the classifiers. Therefore, effective binary code templates are critical for whole method.

For each subject, k class-neighborhood locations are determined by the subject relationship matrix, and we generate binary code templates according to $\mathrm{k}$ class-neighborhood locations. Equation (1) shows the transform form. Information entropy of binary code templates is considered to determine the value of $\mathrm{k}$, and information entropy is obtained by (2).

$$
L_{i j} \begin{cases}1 & \text { if } j \text { is } k \text { class }- \text { neighborhood of } i \\ 0 & \text { others }\end{cases}
$$




$$
\begin{aligned}
& \text { Ent }=-\left(p\left(l_{i}(a=0)\right) \log p\left(l_{i}(a=0)\right)\right. \\
& \left.-p\left(l_{i}(a=1)\right) \log p\left(l_{i}(a=1)\right)\right)
\end{aligned}
$$

In (2), $p\left(l_{i}(a=0)\right)$ is the probability that a bit value is 1 in the i-th binary code template. The following is a binary code template:

$$
l_{i}=010010101 \text {, then: } p\left(l_{i}(a=0)\right)=\frac{5}{9}, p\left(l_{i}(a=1)\right)=\frac{4}{9}
$$

\section{Binary Code Learner Training}

Taking a binary codes learner training with 4 subjects (A, B, $\mathrm{C}, \mathrm{D})$ as an example, through the previous two steps, we can obtain the binary code templates shown in Figure 4. When we train the i-th bit(i=1,2,3,4), all the training samples in subject $\mathrm{j}(\mathrm{j}=1,2,3,4)$ are labeled as $L_{i j}$, it's easy to know that, when $\mathrm{i}=\mathrm{j}$, $L_{i j}$ always equal to 1 . The i-th SVM is trained with all training samples and their supervised information as input. In this example, we need to train 4 SVM models.

$\begin{array}{ccccc} & \text { Bit1 } & \text { Bit2 } & \text { Bit3 } & \text { Bit4 } \\ \text { Template A } & 1 & 0 & 1 & 1 \\ \text { Template B } & 0 & 1 & 0 & 0 \\ \text { Template C } & 1 & 0 & 1 & 1 \\ \text { Template D } & 1 & 0 & 1 & 1\end{array}$

FIGURE IV. AN EXAMPLE OF BINARY CODES LEARNER TRAINING.

\section{The Flow of BCLCN for Finger Vein Recognition}

The flow of BCLCN for finger vein recognition is show in Figure 5. The whole method is divided into training phase and testing phase. In addition, to reduce the variations of image localization, Region of Interest (ROI) of original image is determined in both of training phase and test phase[10]. In order to improve the reliability of the subject relationship matrix, moreover, to reduce the computational cost, we obtain the histogram of oriented lines(HOL) [11] features of all finger vain $\mathrm{ROI}$ images first.

In training phase, based on HOL features, subject relationship matrix is constructed. After that, relationship matrix is transformed into binary code templates according to $\mathrm{k}$ class-neighborhood locations. At last, SVM models are trained for bits with all training samples and their supervised information as input. The detailed procedures are shown in the sections above.

In testing phase, each test HOL feature is input into the SVM model to generate binary code in turn. The verification result can be obtained by calculating Hamming distance between the test binary code and the binary code templates.

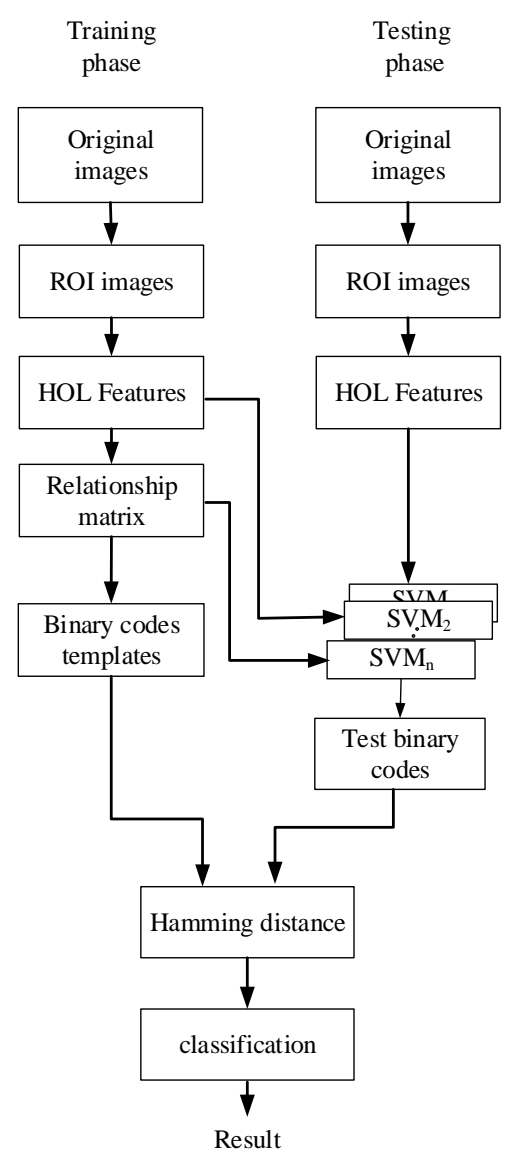

FIGURE V. THE FLOW OF PROPOSED METHOD.

\section{EXPERIMENTAL AND RESULT ANALYSIS}

\section{A. Databases}

In this paper, self-built finger vein database is used for the performance evaluation of the proposed method. self-built finger vein database consists of 2610 finger vein images captured from 29 volunteers. Each person was asked to provide images from the left hand to right hand, index, middle, index fingers respectively, 6 fingers per person, 15 images per finger, each finger is considered as an individual. The image size is $640 \times 480$ pixels. The localized ROI image has the pixel size of 130 pixels $\times 370$ pixels. The first 5 samples of each subject are used for constructing training set and the left 10 samples of each subject are used for testing. To this end, the equal error rate (EER), which is the point where the false accept rate (FAR) is equal to the false reject rate (FRR) is adopted to be the evaluation criterion.

\section{B. Experimental}

Because the value of k class-neighborhood directly affects the presentation ability of the binary templates, so the first experiment is to investigate the optimal $\mathrm{k}$ value. Figure 6 depicts the information entropy value (Ent) of single template according to different $\mathrm{k}$, and the change of Ent is a symmetric curve. Single template has 174 bits in our experiment (training 
set have 174 subjects), and when $\mathrm{k}=87$, Ent is maximum 0.69 , with increasing or decreasing the value of $\mathrm{k}$, Ent is decreased. We change $\mathrm{k}$ from 35 to 139(according to Ent $>0.5$ ), EERs are listed in Table 1. As show in the table, taking into consideration of matching accuracy and information entropy, $\mathrm{k}$ is selected as 60 , and the best EER $=0.512 \%$.

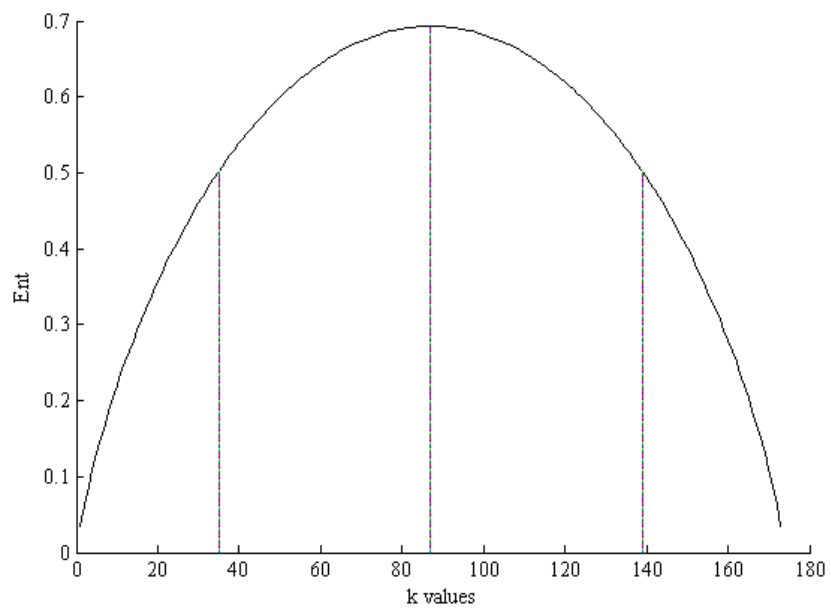

FIGURE VI. ENT OF SINGLE TEMPLATE ACCORDING TO DIFFERENT K

TABLE I. EER VALUES ACCORDING TO THE NUMBER OF THE VALUES OF K CLASS-NEIGHBORHOOD

\begin{tabular}{cc|cc}
\hline $\mathbf{k}$ & $\mathbf{E E R / \%}$ & $\mathbf{k}$ & $\mathbf{E E R / \%}$ \\
\hline 35 & 0.653 & 90 & 0.610 \\
45 & 0.560 & 100 & 0.556 \\
50 & 0.553 & 110 & 0.629 \\
55 & 0.787 & 120 & 0.729 \\
60 & 0.512 & 130 & 0.713 \\
70 & 0.584 & 139 & 0.643 \\
80 & 0.606 & & \\
\hline
\end{tabular}

To verify the validity of the proposed method, in this experiment, state-of-the-art methods for finger vein recognition such as LBP[6], (2D) ${ }^{2} \mathrm{FPCA}[12]$, BLPOC[7], Gabor[4], HOG[8] and HOL[11]are implemented for comparison. Table 2 shows the receiver operating characteristics (ROC) curves derived from the above methods. We can observe that the proposed method outperforms the other methods in performance. For other methods, as a traditional binary code, LBP do not perform well in finger vein recognition, because it is just binary based pixels. Gabor filter can obtain the line responses and orientation of pixels in finger vein images. HOL is a variation of HOG, and the calculation method of gradient amplitude and direction in HOG is improved by Gabor filter, the result proves its effectiveness for finger vein recognition. Proposed method constructs the subject relationship matrix based on HOL, HOL feature can guarantee the reliability of the subject relationship, meanwhile, the discriminative information among different classes is used for describing the subject, these properties ensure the effectiveness of the proposed method.

TABLE II. EER VALUES OF DIFFERENT METHODS

\begin{tabular}{cl}
\hline Methods & EER/\% \\
\hline LBP & 4.200 \\
$(2 \mathrm{D})^{2}$ FPCA & 3.735 \\
BLPOC & 2.348 \\
Gabor & 1.667 \\
HOG & 0.804 \\
HOL & 0.683 \\
Proposed method & 0.512 \\
\hline
\end{tabular}

\section{CONCLUSION}

This paper presents a novel binary code learning method based on class-neighborhood for finger vein recognition. The proposed method BCLCN has three main steps: subject relationship matrix construction; binary code templates generation and binary code learner training. In the first step, to reduce the length of binary codes, the centers of subjects are used to compute the between-class correlation. After that, histogram equalization is used to amplify the difference of correlation in original relationship matrix. In the second step, for each subject, the binary code template according to k classneighborhood locations is generated. To ensure all templates are discriminative and informative, information entropy of binary code templates is considered to determine the value of k. Finally, SVMs are trained as code learner for each bit, with all training samples as input and binary code templates as supervised information. Through theoretical analysis and experiments, the feasibility of BCLCN is validated.

\section{ACKNOWLEDGMENT}

This research was financially supported by the National Natural Science Foundation of China, and the Grant Number is 61572458.

\section{REFERENCES}

[1] Hashimoto J. Finger vein authentication technology and its future[C] //Proceedings of the Symposium on VLSI Circuits. Los Alamitos: IEEE Computer Society Press, 2006: 5-8.

[2] Kumar A, Zhou Y. Human identification using finger images[J], IEEE Trans. Image Process. 21 (4) (2012) 2228-2244.

[3] Miura N, Nagasaka A, Miyatake T. Feature extraction of finger- vein patterns based on repeated line tracking and its application to personal identification[J]. Machine Vision and Applications, 2004, 15(4): 194203.

[4] Xie S J, Yang J C, Yoon S, Lu Y, Park D S. Guided Gabor filter for finger vein pattern extraction[C] //Proceedings of the 8th International Conference on Signal Image Technology and Internet Based Systems. Los Alamitos: IEEE Computer Society Press, 2012: 118-123.

[5] [5] Khellat-Kihel S, Abrishambaf R, Cardoso N, Monteiro J, Benyettou M. Finger vein recognition using Gabor filter and support vector machine[C] //Proceedings of the International Image Processing Applications and Systems Conference. Los Alamitos: IEEE Computer Society Press, 2014: 1-6.

[6] Lee E C, Lee H C, Park K R. Finger vein recognition using minutiabased alignment and local binary pattern-based feature extraction[J]. 
International Journal of Imaging Systems and Technology, 2009, 19(3): 179-186.

[7] Mahri N, Suandi S A S, Rosdi B A. Finger vein recognition algorithm using phase only correlation[C] //Proceedings of the International Workshop on Emerging Techniques and Challenges for Hand-Based Biometrics. Los Alamitos: IEEE Computer Society Press, 2010: 1-6.

[8] Dalal N, Triggs B. Histograms of oriented gradients for human detection[C] //Proceedings of IEEE Computer Society Conference on Computer Vision \& Pattern Recognition. Los Alamitos: IEEE Computer Society Press, 2005, 1: 886-893.

[9] Xi X M, Yang L, Yin Y. Learning discriminative binary codes for finger vein recognition[J]. Pattern Recognition, 2017, 66:26-33.

[10] Zhang L P, Li W J, Ning X, Dong X L, Liu W J. A Finger Vein Recognition Method Based on Histogram of Oriented Lines and (2D)2FPCA[J]. Journal of Computer-Aided Design \& Computer Graphics, 2018, 30(2): 254-261.

[11] Jia W, Hu R X, Lei Y K, Zhao Y, Gui J. Histogram of oriented lines for palmprint recognition[J]. IEEE Transactions on Systems Man, \& Cybernetics: Systems, 2014, 44(3): 385-395.

[12] Wang J, Li H J, Wang G Q, Li M, Li D. Vein recognition based on (2D)2FPCA[J]. International Journal of Signal Processing, Image Processing and Pattern Recognition, 2013, 6(4): 323-332. 